\title{
Consulta pré-operatória de enfermagem e o autocuidado do paciente oncológico com estomia respiratória
}

\author{
Preoperative nursing consultation and self-care of cancer patients with \\ respiratory ostomy
}

\section{Consulta de enfermería preoperatoria y autocuidado de pacientes oncológicos con estoma respiratório}

Renata Otoni Neiva ${ }^{1, *}$, Márcio Corrêa Nogueira² ${ }^{2}$ Adriana Jimenez Pereira ${ }^{3}$

\section{ORCID IDS}

Neiva RO (D) https://orcid.org/0000-0001-9622-5711

Nogueira MC (D) https://orcid.org/0000-0001-7964-632X

Pereira AJ (D) https://orcid.org/0000-0002-5348-3945

\section{COMO CITAR}

Neiva RO; Nogueira MC; Pereira AJ. Consulta pré-operatória de enfermagem e o autocuidado do paciente oncológico com estomia respiratória. ESTIMA, Braz. J. Enterostomal Ther., 2020, 18: e2920. https://doi.org/10.30886/estima.v18.914_PT

\section{RESUMO}

Objetivo: demonstrar a influência da consulta pré-operatória de enfermagem na prática do autocuidado realizado pelos pacientes com estomia respiratória do A.C. Camargo Câncer Center e descrever quais são as práticas de autocuidado realizadas e seus fatores limitadores. Método: estudo qualitativo, exploratório, realizado com 7 pacientes com idade igual ou superior a 18 anos, submetidos à consulta pré-operatória de enfermagem, que realizaram cirurgia com confecção de estomia respiratória e estavam com capacidade de estabelecer comunicação verbal. Os dados foram coletados mediante entrevista semiestruturada e gravador digital e submetidos à análise de conteúdo. Resultados: predominou participantes homens, com idade igual ou superior a 56 anos e $3^{\circ}$ grau completo. Foram identificadas duas categorias analíticas: "Repercussões das ações promovidas pela consulta pré-operatória de enfermagem"; e "Obstáculos na busca pela autonomia no cuidado com a cânula de traqueostomia". Conclusão: a consulta de enfermagem é um importante instrumento para orientar, formar vínculo e esclarecer dúvidas sobre os cuidados com a estomia e dispositivos respiratórios, e as ações de enfermagem podem limitar e até impedir o paciente de desenvolver o seu autocuidado, fazendo-se necessário que o projeto terapêutico de enfermagem seja planejado para agregar o paciente nesse processo.

DESCRITORES: Enfermagem no consultório; Enfermagem oncológica; Autocuidado; Estomas cirúrgicos; Traqueostomia; Estomaterapia.

\footnotetext{
1. A.C. Camargo Câncer Center - Departamento de Cabeça e Pescoço - Ambulatório de Curativos - São Paulo (SP), Brasil.

2. Centro Universitário São Camilo - Departamento de Pós-Graduação Lato Sensu Enfermagem em Estomaterapia - São Paulo (SP), Brasil.

3. Centro Universitário São Camilo - Departamento de Enfermagem - São Paulo (SP), Brasil.
}

*Autora correspondente: renata_otoni@hotmail.com

Recebido: Jul. 01, 2020 | Aceito: Nov. 11, 2020 


\begin{abstract}
Objective: to demonstrate the influence of the preoperative nursing consultation in the practice of self-care performed by patients with respiratory ostomy at the A.C. Camargo Cancer Center and to describe what are the self-care practices performed and their limiting factors. Method: qualitative, exploratory study, carried out with 7 patients aged 18 years or older, who underwent preoperative nursing consultation, who underwent surgery with the manufacture of a respiratory ostomy and were able to establish verbal communication. Data was collected through semi-structured interviews and a digital recorder and submitted to content analysis. Results: male participants predominated, aged 56 years or over and completed high school. Two analytical categories were identified: "Repercussions of the actions promoted by the preoperative nursing consultation"; and "Obstacles in the search for autonomy in the care of the tracheostomy cannula". Conclusion: nursing consultation is an important instrument to guide, form bonds and clarify doubts about care with ostomy and respiratory devices, and nursing actions can limit and even prevent the patients from developing their self-care, making it necessary that the therapeutic nursing project is planned to aggregate the patient in this process.
\end{abstract}

DESCRIPTORS: Nursing in the office; Oncology nursing; Self-care; Surgical stomas; Tracheostomy; Stomatherapy.

\title{
RESUMEN
}

Objetivo: demostrar la influencia de la consulta preoperatoria enfermería en la práctica del autocuidado realizado por pacientes con estoma respiratoria en el AC Camargo Cancer Center, describa qué prácticas de autocuidado se realizan y sus factores limitantes. Método: estudio cualitativo, exploratorio, realizado con 7 pacientes de 18 años o más, que fueron intervenidos de consulta de enfermería preoperatoria, quienes fueron intervenidos con la fabricación de un estoma respiratorio, y pudieron establecer comunicación verbal. Los datos se recopilaron mediante entrevistas semiestructuradas y una grabadora digital, y se sometieron a análisis de contenido. Resultados: predominó el sexo masculino, de 56 años o más, y bachillerato completo. Se identificaron dos categorías analíticas: "Repercusiones de las acciones promovidas por la consulta de enfermería preoperatoria"; y "Obstáculos en la búsqueda de autonomía en el cuidado de la cánula de traqueotomía". Conclusión: la consulta de enfermería es una herramienta importante para orientar, vincular y aclarar dudas sobre el cuidado con el estoma y los dispositivos respiratorios, y las acciones de enfermería pueden limitar e incluso impedir que el paciente desarrolle su autocuidado, haciéndolo necesario que el proyecto de enfermería terapéutica está planificado para agregar al paciente en este proceso.

DESCRIPTORES: Consulta de enfermería; Enfermería oncológica; Cuidados personales; Estomas quirúrgicos; Traqueotomía; Estomaterapia.

\section{INTRODUÇÃO}

O câncer de cabeça e pescoço compreende um grupo de neoplasias malignas que tem origem na cavidade oral, orofaringe, laringe e hipofaringe, sendo descrito frequentemente como "carcinoma de células escamosas do trato aerodigestivo superior (UADT)"1.

Mundialmente, é o $6^{\circ}$ tipo de câncer mais prevalente com 9,2\% dos casos, sendo responsável por 4,6\% das mortes relacionadas ao câncer. No Brasil, está associado a uma alta mortalidade, ocupando o quinto lugar na causa de morte. $\mathrm{O}$ Instituto Nacional do Câncer (INCA) estimou para o ano de 2020, no Brasil, o surgimento de 15.190 casos novos de câncer na cavidade oral, ocorrendo 11.180 casos em homens e 4.010 casos em mulheres ${ }^{1,2}$.

Os fatores de risco mais bem estabelecidos para o desenvolvimento desses tipos de cânceres é o consumo do tabaco associado ou não ao uso do álcool, estando relacionados também outros fatores de risco, como exposição solar sem proteção para câncer de lábio, exposição ocupacional e o vírus do Papiloma Humano (HPV) $)^{1,2}$.
Em mais de 60\% dos pacientes o diagnóstico do câncer de cabeça e pescoço ocorre quando a doença está em estádios clínicos avançados, isso porque os primeiros sintomas apresentados são de natureza inespecífica e, também, pelo fato de que eles podem acessar os canais linfáticos no início do seu aparecimento e se desenvolver rapidamente. Sendo assim, pacientes com doença avançada necessitam de tratamento mais complexo, com associação de diferentes modalidades terapêuticas, como cirurgia, radioterapia e quimioterapia, e consequentemente com maior morbidade e comprometimento da qualidade de vida ${ }^{1,3}$.

Com o objetivo de manter a via aérea pérvia diante do tratamento do câncer de cabeça e pescoço, muitos pacientes necessitaram, temporariamente ou definitivamente, de uma traqueostomia. Esse procedimento consiste em uma abertura na parede anterior da traqueia (estomia respiratória), que se comunica com o meio externo através de uma cânula, gerando mudanças na dinâmica respiratória do indivíduo, bem como em seu comportamento, relacionamento interpessoal e cuidado pessoal ${ }^{4}$.

As necessidades e as reações singulares de um portador de estomia estão subentendidas em sua identidade e subjetividade. Dessa forma, os problemas ocasionados pela abertura de uma estomia estão relacionados com as condições pessoais de cada 
indivíduo, incluindo a qualidade do suporte assistencial recebido nas fases do tratamento cirúrgico gerador da estomia. Nesse contexto, destaca-se a consulta de enfermagem (CE), uma atividade privativa do enfermeiro, respaldada por lei, relevante e resolutiva, que identifica situações de saúde/doença, prescreve e executa ações de enfermagem que contribuem para a promoção da saúde, prevenção e proteção de agravos, além da recuperação e reabilitação do indivíduo, família e comunidade ${ }^{5}$.

A consulta de enfermagem que antecede o procedimento gerador de uma estomia respiratória é uma importante ferramenta terapêutica, que proporciona ao paciente um espaço para esclarecimento de suas dúvidas e/ou dos seus familiares. Dentro do seu escopo de atuação, neste momento, o enfermeiro traz informações sobre o procedimento cirúrgico, a estomia respiratória e seus dispositivos e outros temas mais que julgue necessário, visando reduzir as complicações cirúrgicas imediatas, estabelecer vínculo e identificar problemas, além de proporcionar uma oportunidade de educação em saúde precoce sobre a importância do autocuidado no período pós-operatório, com uma linguagem acessível.

Justifica-se este estudo pela escassez de pesquisas relacionadas à consulta pré-operatória de enfermagem na promoção do autocuidado do paciente oncológico com a estomia respiratória. Os questionamentos foram os seguintes: a consulta pré-operatória de enfermagem contribui para o desenvolvimento do autocuidado do paciente oncológico com a estomia respiratória? Quais são as práticas de autocuidado realizadas por estes pacientes?

\section{OBJETIVO}

Demonstrar a influência da consulta pré-operatória de enfermagem na prática do autocuidado realizado pelos pacientes com estomia respiratória pós-cirúrgicas do A.C. Camargo Câncer Center, e descrever, a partir da análise de conteúdo, quais são as práticas realizadas e os fatores limitadores para o desenvolvimento da autonomia no autocuidado.

\section{MÉTODO}

Trata-se de um estudo qualitativo, de caráter exploratório, que foi realizado no Departamento de Cirurgia de Cabeça e Pescoço do A.C. Camargo Câncer Center, um centro oncológico localizado na cidade de São Paulo. O A.C.
Camargo Câncer Center é uma instituição privada, sem fins lucrativos, mantida pela fundação Antônio Prudente, que trata pacientes oncológicos provenientes do Sistema Único de Saúde (SUS), de seguradoras (convênio) e privados (particulares).A presente pesquisa foi conduzida de acordo com os preceitos éticos da Resolução no 466/12 CNS/MS. A coleta foi iniciada após aprovações pelo Comitê de Ética em Pesquisa com Seres Humanos do Centro Universitário São Camilo e pelo comitê de Ética em Pesquisa do A.C. Camargo Câncer Center, sob o número 2635/18.

Os pacientes elegíveis para o estudo foram selecionados no período de fevereiro/2018 a abril/2018. Foram incluídos 7 pacientes com idade igual ou superior a 18 anos, que foram submetidos no pré-operatório à consulta de enfermagem estabelecida pela instituição, que estavam no período pósoperatório de cirurgias de cabeça e pescoço avaliadas como de grande porte (ressecção de tumores de base e/ou língua e outros tumores em região orofaríngea), que necessitaram de estomia respiratória transitória e que se encontravam em processo de reabilitação ambulatorial apresentando capacidade de estabelecer comunicação verbal. Foram excluídos os pacientes laringectomizados totais por ainda não estarem reabilitados para comunicação verbal e os que tiveram reversão da estomia respiratória antes do sétimo dia de pós-operatório, por acreditar que período inferior a esse seria insuficiente para desenvolver o autocuidado.

Inicialmente, foi acessado um documento de registro de todos os pacientes que realizaram a consulta de enfermagem pré-operatória no departamento de cabeça e pescoço. Após isso, através da plataforma $\mathrm{MV}$, sistema informatizado utilizado para registro dos prontuários dos pacientes no A.C. Camargo Câncer Center, as informações desses pacientes foram consultadas, e selecionados aqueles elegíveis de acordo com os critérios de inclusão. Foi investigada a programação de seus retornos ambulatoriais de acordo com a agenda de consultas e procedimentos. Os pacientes selecionados e que compareceram, conforme programado, foram abordados após a realização dos procedimentos agendados e convidados a participarem da pesquisa, sendo informados sobre o contexto, métodos da pesquisa e os seus riscos. Dessa forma, após o esclarecimento sobre o estudo, foi solicitado a eles a leitura e a assinatura do Termo de Consentimento Livre e Esclarecido (TCLE) em duas vias, para que confirmassem as informações e concordassem em participar. Sendo uma das vias entregue ao participante e a outra arquivada pelo pesquisador. Todos os pacientes convidados aceitaram participar da pesquisa. 
Após assinatura do termo de consentimento, o paciente foi atendido em ambiente adequado, acompanhado por seu familiar/cuidador e pela pesquisadora para a aplicação de um questionário específico, semiestruturado, composto por perguntas abertas e fechadas, contemplando o objetivo do estudo. Além disso, foi utilizado um gravador digital para facilitar a posterior transcrição e análise do seu conteúdo. As entrevistas duraram em média 30 minutos.

As transcrições foram realizadas posteriormente, sendo necessárias repetições dos áudios de três entrevistas, pois a dicção desses participantes estava comprometida pelo procedimento cirúrgico. Os dados foram discutidos e trabalhados a partir da análise de conteúdo. Tal técnica consiste em interpretar o significado do que é falado sobre um determinado assunto por meio da categorização do conteúdo dos relatos. O conteúdo foi analisado de acordo com as seguintes fases: pré-análise - com a escolha e organização do material a ser analisado e formulação das hipóteses; exploração - que consiste na transformação dos dados e unidades de registro em unidades de contexto; e análise - com o tratamento dos resultados, interpretações e conclusões ${ }^{6}$.

\section{RESULTADOS}

A amostra deste estudo foi composta por sete participantes, cuja caracterização está demonstrada na Tabela 1.

Tabela 1. Caracterização do perfil da amostra. Botucatu (SP), Brasil - 2019.

\begin{tabular}{|c|c|c|}
\hline Sexo & $\begin{array}{l}\text { Número/ } \\
\text { Idade }\end{array}$ & $\begin{array}{l}\text { Mediana/ } \\
\text { Fração \% }\end{array}$ \\
\hline Masculino & 5 & $71 \%$ \\
\hline Feminino & 2 & $29 \%$ \\
\hline Idade & $\begin{array}{l}\text { Número/ } \\
\text { Idade }\end{array}$ & $\begin{array}{l}\text { Mediana/ } \\
\text { Fração \% }\end{array}$ \\
\hline E1 & 38 & \\
\hline E2 & 19 & \\
\hline E3 & 68 & \\
\hline $\mathrm{E} 4$ & 57 & 47 anos \\
\hline E5 & 55 & \\
\hline E6 & 57 & \\
\hline E7 & 36 & \\
\hline $\begin{array}{c}\text { Tempo de } \\
\text { permanência da } \\
\text { estomia respiratória }\end{array}$ & $\begin{array}{l}\text { Número/ } \\
\text { Idade }\end{array}$ & $\begin{array}{l}\text { Mediana/ } \\
\text { Fração \% }\end{array}$ \\
\hline 7 a 14 dias & 3 & $43 \%$ \\
\hline 15 a 21 dias & 2 & $28,5 \%$ \\
\hline 22 a 28 dias & 2 & $28,5 \%$ \\
\hline
\end{tabular}

Após a leitura dos discursos coletados nas entrevistas, foram identificadas duas categorias analíticas: "Repercussões das ações promovidas pela consulta de enfermagem préoperatória" e "Obstáculos na busca pela autonomia no cuidado com a cânula de traqueostomia”.

\section{Repercussões das ações promovidas pela consulta de enfermagem pré-operatória}

A consulta pré-operatória de enfermagem para pacientes do departamento de cabeça e pescoço foi implementada na instituição em novembro de 2017. Nessa abordagem as enfermeiras estomaterapeutas que atuam no setor apresentam para os pacientes e familiares, dentre outros assuntos, a definição de traqueostomia, estomia respiratória e seus dispositivos. Como instrumentos facilitadores da prática de educação em saúde desenvolvida são utilizados folhetos informativos com os tipos de cânulas de traqueostomia utilizadas na instituição e demonstrado o seu manuseio. São abordados também os cuidados necessários com o dispositivo, tais como: a higiene do intermediário ou endocânula, a substituição dos curativos para traqueostomia e fixadores descartáveis.

“(...) A questão da limpeza né? Então, lá no quarto eu já pedia pra limpar porque eu sabia que precisava né?”. E1

“(...) Quanto à higienização, o que tinha que ser feito. Tirar a cânula pra lavar, higienizar né? Então tudo o que foi me passado eu fiquei atenta, mas foi tudo feito mesmo de acordo". E5

“(...) Me explicou toda a parte de como manusear a cânula. Mostrou os tipos de cânulas, a evolução que teria né, ir trocando uma pela outra. Foi o que ocorreu. Inclusive me explicou quais seriam os pós, se eu saísse daqui tinha até uma proteção que poderia ser colocada. Me mostrou o aparelho também”. E6

A cirurgia de cabeça e pescoço com confecção de estomia respiratória determina alterações psicológicas e funcionais, como na deglutição e fala, muito significativas para o paciente, que podem acarretar sequelas físicas, emocionais e sociais. É o início de um processo de dependência da cânula de traqueostomia, uma condição que pode gerar insegurança, medo e constrangimentos pela evidência do dispositivo e presença de secreções. Fazem-se necessárias ações de educação e orientação que preparem esse indivíduo 
para o enfrentamento dessa nova condição, oferecendo a ele informações e conhecimentos para adquirir habilidades e desenvolver o seu autocuidado.

Nesse contexto, observa-se a relevância do enfermeiro como educador no período pré-operatório. Verifica-se nas falas que as orientações fornecidas contribuíram para uma melhor percepção e preparo dos pacientes.

“(...) Ah, eu não conhecia nada né? Então já deu uma boa ideia do que ia ser". E1

“(...) eu fui muito bem atendido, muito bem assistido. Neste ponto vocês fizeram bastante, só tenho o que agradecer. Foram muito importantes (as informações)”. E3

“(...) Tudo foi dentro do que foi me dito, e foi bom. Porque você já sabe do caminho que você vai enfrentar”. E4

“(...) Os cuidados que tinha que ser feitos, tudo foi me passado antes". E5

“(...) eu consegui assimilar tudo porque vocês falaram”. E7

Algumas entrevistas apresentaram também as dificuldades que os pacientes enfrentaram nesse processo do cuidado e mencionaram que alguns fatores não foram abordados na consulta de enfermagem pré-operatória:

“(...) Só que ficava muito difícil de respirar um pouco. É quando vai limpar, aí tira e joga água, eu me sentia afogado (aspiração traqueal)”. E2

“(...) o maior problema da cânula pra mim foi a primeira vez que ela ficou presa com um ponto, então ela me causava uma ferida que doía muito”. E4

“(...) eu só acho que deviam falar mais pra gente quando tira a cânula, da necessidade de sempre apertar quando for tossir, falar, porque eu tive uma dificuldade muito grande de fechar". E4

Em cirurgias de cabeça e pescoço com reconstrução microcirúrgica, as cânulas de traqueostomia são fixadas na pele do paciente com pontos de sutura por um período pré-determinado. O objetivo dessa abordagem é prevenir complicações com o retalho cirúrgico. Após a decanulação, é realizada a aproximação das bordas da estomia com fita adesiva e posterior oclusão com curativo compressivo para favorecer a cicatrização.

Foi possível também notar que a nova condição de saúde, ao impactar emocionalmente os pacientes, desperta sentimentos conflituosos e que influenciam na compreensão das orientações realizadas pela enfermeira no processo educativo, conforme descrito no discurso a seguir:
"(...) é, deu uma visão, é bom que eu já esperava. Eu só lembrei que não ia ter voz, o resto eu não prestei muita atenção não". E2

\section{Obstáculos na busca pela autonomia no cuidado com a cânula de traqueostomia}

Os entrevistados em sua maioria relataram que os cuidados com a cânula de traqueostomia eram realizados pela equipe de enfermagem e/ou familiares, conforme evidenciado nos seguintes trechos das entrevistas:

“(...) eu estava com minha irmã. Às vezes ela ou o enfermeiro (realizava os cuidados com o estoma)”. E1

“(...) não era eu que fazia, era a enfermagem. Não cheguei a fazer a manutenção sozinho, estava sempre saindo secreção, eu sempre chamava alguém para fazer”. E3

“(...) era quando eu estava no hospital e eles faziam no hospital. Então todo o processo foi com os enfermeiros, a higiene era por conta deles. Eu só falava quando estava incomodando né, aí eles tiravam e limpavam, a higienização era por conta deles. Não fui eu que fiz, eram os enfermeiros mesmo. Eu estava internada e tudo...”. E5

“(...) eu não mexia nele, eu não coloquei a mão no meu estoma. Quem fazia eram as enfermeiras, ou era ele (marido)". E7

A presença de uma estomia respiratória modifica a anatomia e fisiologia respiratória do indivíduo, exigindo novas competências para desenvolver o autocuidado. Verificou-se que com o objetivo de realizar a assistência e apoiar a participação familiar nos cuidados aos pacientes, os profissionais correm o risco de possibilitar uma dependência, comprometendo o desenvolvimento do autocuidado do indivíduo com a nova condição de saúde.

É importante que para o desenvolvimento do autocuidado o paciente demonstre determinação e desejo em realizá-lo. Nesse cenário, é fundamental o trabalho educativo da enfermagem para encorajar, estimular e aprimorar as habilidades do paciente na conquista da sua autonomia.

Nas situações em que os entrevistados realizaram cuidados com a cânula de traqueostomia, essas atividades foram compartilhadas com a enfermagem.

“(...) quando eu consegui tirar (o intermediário), é, consegui mas não sozinho né? Tinha a enfermeira do lado. Oh, aquela 
que segurava assim, eu conseguia trocar, ou a enfermeira trocava e eu segurava”. E2

“(...) a manoplinha, aquela que sai né? Conseguia tirar fora e fazer uma limpeza, durante a internação, mas na maioria das vezes a própria enfermagem que fez né?”. E6

No único relato sobre a realização do autocuidado com autonomia, identificou-se que já havia uma experiência anterior do paciente com o uso da cânula de traqueostomia, fato que contribuiu para uma maior segurança e habilidade para manuseio do dispositivo.

“(...) eu fazia a limpeza sozinho. Eu conseguia abrir, tirar e limpar. Eu olhava no espelho e limpava o que era possível”. E4

\section{DISCUSSÃO}

Quanto ao perfil dos participantes, evidenciou-se que o maior contingente é composto por pacientes do sexo masculino, com idade igual ou superior a 56 anos. Os dados encontrados corroboram os estudos existentes na literatura que evidenciam maior incidência do câncer de cabeça e pescoço em homens. Geralmente estes indivíduos estão entre 50 e 60 anos de idade, embora nos cânceres associados ao vírus HPV, é possível observar uma redução na idade ${ }^{1,3,7}$.

Ao que se refere a limitações da pesquisa, pode-se destacar, como principal, a quantidade de participantes que foi inviabilizada pelo limite temporal estabelecido para a coleta de dados e pela complexidade das cirurgias de cabeça e pescoço consideradas de grande porte, que limitou a participação de pacientes devido os critérios de inclusão estabelecidos neste estudo. Considerando o tamanho da amostra, os resultados devem ser observados com cautela. Entretanto, os achados encontrados neste estudo sobre o autocuidado com a estomia respiratória apoiam e encontra-se em concordância com a pouca literatura identificada sobre a temática.

$\mathrm{Na}$ categoria "Repercussões das ações promovidas pela consulta pré-operatória de enfermagem”, os discursos dos pacientes centraram a abordagem sobre a relevância da consulta de enfermagem e reforçaram a importância das orientações fornecidas, que possibilitaram conhecimento sobre a estomia respiratória e os cuidados necessários, como a higiene da cânula de traqueostomia e segurança sobre a manipulação de tais dispositivos. Esses resultados evidenciam a consulta pré-operatória de enfermagem como relevante instrumento de trabalho do enfermeiro, ressaltando o seu papel como educador em saúde.

Sabe-se que a condição de ser portador de uma estomia respiratória altera a percepção da imagem corporal do paciente e gera mudanças no seu estilo de vida e no desenvolvimento de suas atividades diárias. Essas percepções e os mecanismos de enfrentamento perante a nova condição de saúde são fatores que estão condicionados à cultura, idade, qualidade do suporte familiar e assistencial recebidos em todas as fases do tratamento ${ }^{8}$.

$\mathrm{Na}$ categoria "Obstáculos na busca pela autonomia no cuidado com a cânula de traqueostomia", observou-se que a maioria dos pacientes referia que os cuidados com a estomia respiratória eram realizados pela equipe de enfermagem e/ ou cuidadores. Nesse contexto, o enfermeiro é capacitado e responsável por avaliar corretamente o paciente e realizar a sistematização da assistência de enfermagem (SAE), administrando possíveis riscos assistenciais e gerenciando os cuidados e a assistência da equipe de enfermagem. $\mathrm{O}$ plano terapêutico consiste na limpeza da pele periestoma, na manutenção e troca dos curativos para traqueostomia, assim como a substituição do conjunto de cânulas, higiene da endocânula e aspiração de secreção traqueal quando necessário, a fim de evitar o surgimento de lesões e assegurar um ambiente seguro e livre de contaminação?.

Assim, os cuidados dispensados aos pacientes são indiscutivelmente importantes, mas alguns deles devem ser transferidos gradativamente à pessoa com estomia para que o sujeito adquira segurança e desenvolva competências e habilidades para realizar seu autocuidado, retomando suas atividades diárias e contribuindo para uma melhor qualidade de vida. São os obstáculos que as pessoas com estomia encontram para estabelecer o autocuidado com a estomia que podem determinar uma dependência de seus familiares e/ou profissionais de saúde ${ }^{10}$.

O ensino do autocuidado deve ser iniciado no período pré-operatório, logo após a decisão sobre a abordagem cirúrgica que será realizada. O enfermeiro deve utilizar recursos de ensino-aprendizagem para estabelecer vínculo com paciente/cuidador para facilitar um melhor entendimento sobre as alterações físicas e funcionais decorrentes da confecção de uma estomia respiratória. No pós-operatório os cuidados são com a estomia, a pele periestoma, limpeza e troca dos dispositivos, sendo o paciente o protagonista neste cenário em que ele deverá 
esclarecer dúvidas e demonstrar suas habilidades para o autocuidado domiciliar. Nesse período ele entende melhor as informações fornecidas no pré-operatório, o que poderá contribuir para seu ajuste físico e psicológico, melhor recuperação, adequação e sucesso no autocuidado. Essas orientações devem ser contínuas e centralizadas nas dificuldades dos pacientes e seus familiares/cuidadores ${ }^{11}$.

Através do vínculo estabelecido com o paciente, o enfermeiro pode conhecer quais são as dificuldades encontradas para prática do autocuidado, podendo delimitar, dentro de seu escopo de atuação, medidas e ações de saúde a fim de atender suas necessidades.

Cabe também ao enfermeiro, dentro desse contexto, avaliar o paciente quanto ao desenvolvimento das competências necessárias para o manejo da estomia e dos dispositivos respiratório. Dessa forma, é importante delimitar as competências a serem desenvolvidas a curto, médio e longo prazo para que o profissional possa diagnosticar corretamente em qual etapa o paciente se encontra e através de uma avaliação delimitar novos objetivos a serem alcançados ou readequar e redirecionar os objetivos anteriores ao paciente durante $o$ processo de construção do autocuidado. Ao desempenhar o autocuidado com segurança o paciente ganha autonomia e se organiza em sua rotina diária ${ }^{12}$.

Outro fator observado ainda na categoria "Obstáculos na busca pela autonomia no cuidado com a cânula de traqueostomia” e que está relacionado intrinsecamente ao desenvolvimento do autocuidado é quanto ao período de permanência da estomia respiratória. Com a progressão no processo de reabilitação, o paciente evolui com mecânica respiratória e deglutição adequada, sem obstrução de vias aéreas superiores e secreções controladas, iniciando-se, então, a decanulação. $\mathrm{O}$ processo de decanulação pode ser variável, de acordo com rotinas estabelecidas em diferentes serviços, mas geralmente se inicia com o desinflar do balonete, e se o paciente apresentar boa tolerância ocorre a substituição da cânula plástica por uma metálica até a retirada da cânula de traqueostomia e realização de um curativo oclusivo da estomia ${ }^{13,14}$.

Indivíduos com estomias provisórias/temporárias se envolvem menos no autocuidado, pois usam mecanismos de fuga e negação como estratégias de enfrentamento diante da estomia. Já indivíduos com estomias respiratórias definitivas desenvolvem competências de autocuidado mais prematuramente por usarem estratégias confrontativas perante a sua nova condição de saúde. Destaca-se que, dentre outros fatores, o tipo de estomia quanto à duração (temporária/ definitiva) e o tempo decorrido da cirurgia podem relacionar a adaptação psicológica à estomia com o desenvolvimento da competência do autocuidado ${ }^{15}$.

\section{CONCLUSÃO}

A confecção da estomia respiratória é um recurso terapêutico bastante utilizado a fim de manter a funcionalidade respiratória de pacientes com câncer e que são submetidos a grandes cirurgias de cabeça e pescoço. Este estudou demonstrou a importância da consulta de enfermagem préoperatória como instrumento para orientação, formação de vinculo e como um importante espaço para os pacientes e familiares sanarem eventuais dúvidas e anseios quanto ao processo de cuidados com a estomia e dispositivos respiratórios, bem como promoção do autocuidado.

Observou-se que as ações de enfermagem podem limitar e até impedir o paciente de desenvolver e se apropriar do seu autocuidado, pois ao realizarem os cuidados com a estomia respiratória sem incluir o indivíduo nesse processo não trabalham a educação em saúde buscando a autonomia dos sujeitos. Esse achado sinaliza a necessidade do enfermeiro em gerenciar a assistência de enfermagem de forma a agregar o paciente ao cuidado, para que esse participe das ações dispensadas à estomia respiratória e seus dispositivos, desenvolvendo competências necessárias para se apropriar do autocuidado.

Com a escassez de conteúdo encontrado durante a construção deste estudo, faz-se necessário o desenvolvimento de um maior contingente de pesquisas nessa temática a fim de proporcionar pesquisas cientificas de qualidade e com importância clínica, que direcione a atuação da enfermagem em todo processo terapêutico do paciente com estomia respiratória, seja através da consulta de enfermagem préoperatória ou durante a sistematização da assistência de enfermagem no período pós-operatório.

\section{CONTRIBUIÇÃO DOS AUTORES}

Conceitualização: Neiva RO, Nogueira MC e Pereira AJ; Metodologia: Neiva RO; Investigação: Neiva RO e Nogueira MC; Redação - Primeira versão: Neiva RO, Nogueira MC e Pereira AJ; Redação - Revisão \& Edição: Neiva RO, Nogueira MC e Pereira AJ; Supervisão: Pereira AJ 


\section{REFERÊNCIAS}

1. Santos FBG, Leonhardt FD, Abrahão M. Prevenção do câncer do trato aerodigestivo superior através das estratégias de busca ativa e uso de propedêutica armada. Braz J Otorhinolaryngol 2020;86(4):443-9. https://doi. org/10.1016/j.bjorl.2019.01.002

2. Santos MO. Estimativa 2018: incidência de câncer no Brasil. Rev bras cancerol 2018;64(1):119-20. Disponível em: https:// www.inca.gov.br/tipos-de-cancer/cancer-de-boca

3. Foreman A, Gullane PJ. Overview of head and necks tumors Plastic surgery. Volume 3. Craniofacial, Head and Necks Surgery and Pediatric Plastic Surgery, 16, 401-426.e2. December 31, 2017.

4. Soares MCCX, Westphal FL, Lima LC, Medeiros JM. Elaboração de protocolo de condutas em traqueostomias no hospital referência de tratamento do câncer do Amazonas. Rev Col Bras Cir 2018;45(4):e1744. https://doi.org/10.1590/01006991 e-20181744

5. Alexandre SG. Construção e validação de instrumentos para consulta de enfermagem à pessoa idosa estomizada fundamentados na teoria do autocuidado [tese]. Fortaleza (CE): Universidade Estadual do Ceará, Centro de Ciência da Saúde; 2017.

6. Silva AH, Fossá MIT. Análise de conteúdo: exemplo de aplicação da técnica para análise de dados qualitativos. Qualitas Revista Eletrônica 2015;17(1). Disponível em: http://revista.uepb.edu.br/index.php/qualitas/article/ view/2113/1403

7. Cocato ACF, Gomes JJ, Biasoli ER, Silva JZ, Miyahara Gl, Neto SC. Fatores de risco da fístula faringocutânea em pacientes laringectomizados. ESTIMA, Braz J Enterostomal Ther 2016; [citado 2020 ago 13]; 13(3). Disponível em: https://www. revistaestima.com.br/estima/article/view/109

8. Souza MMT, Moraes AA, Balbino CM, Silvino ZR, Tavares CMM, Passos JP. Apoio emocional realizado por enfermeiro ao paciente ostomizado. Rev port enferm saúde mental 2016;esp4:49-56. https://doi.org/10.19131/rpesm.0141

9. Oliveira APV, Gomes GC, Romeu BR, Svaldi JSD, Machado GS. Protocolo assistencial de enfermagem a portadores de traqueostomia em ventilação mecânica. HU Revista, Juiz de Fora 2016;42(1):33-41. Disponível em: https://periodicos. ufjf.br/index.php/hurevista/article/view/2353

10. Mota MS, Gomes GC, Silva CD, Gomes VLO, Pelzer MT, Barros EJL. Autocuidado: uma estratégia para a qualidade de vida da pessoa com estomia. Investig Enferm Imagen Desarr 2016;18(1):63-78. https://doi.org/10.11144/Javeriana.ie181.aeqv

11. Lenza NFB, Sonobe HM, Buetto LS, Santos MG, Lima MS. O ensino do autocuidado aos pacientes estomizados e seus familiares: uma revisão integrativa. Rev bras promoç saúde 2013;26(1):139-45. Disponível em: https://www.redalyc.org/ pdf/408/40827988019.pdf

12. Queirós SMM, Santos CSVB, Brito MAC, Pinto IES. Construção do formulário de avaliação da competência de autocuidado na pessoa com ostomia de ventilação. Rev Enf Ref 2015;IV(7):51-60. https://doi.org/10.12707/RIV15010

13. Costa CC, Favero TC, Rosa FB, Steidl EMS, Mancopes R. Decanulação: atuação fonoaudiológica e fisiterapêutica. Distúrb comun 2016;28(1):93-101. Disponível em: https:// revistas.pucsp.br/index.php/dic/article/view/22714/19274

14. Côrte MMD, Vicente LCC, Friche AAL. Decanulação: indicadores sociodemográficos, clínicos e fonoaudiológicos preditivos de sucesso. Audiol Commun Res 2019;24:e2103. https://doi.org/10.1590/2317-6431-2018-2103

15. Queirós SMM, Santos CSVB, Brito MAC, Pinto IES. Factores condicionantes del desarrollo de la competencia de autocuidado en la persona con ostomía de ventilación. Rev Enf Ref 2017;IV(14):57-68. https://doi.org/10.12707/ RIV17010 